OVARIAN CANCER

\title{
Multimodal screening keeping mortality at bay?
}

The poor prognosis and survival outcomes of patients with ovarian cancer have not changed significantly in the past three decades. Most women are diagnosed with advanced-stage disease and large volume metastases at the time of diagnosis; as a result, fewer than $40 \%$ of patients survive beyond 5 years. Women undergo invasive surgical treatment and chemotherapy, sometimes with minimal benefit, which has prompted an effort to focus on earlier detection to ultimately reduce mortality from ovarian cancer.

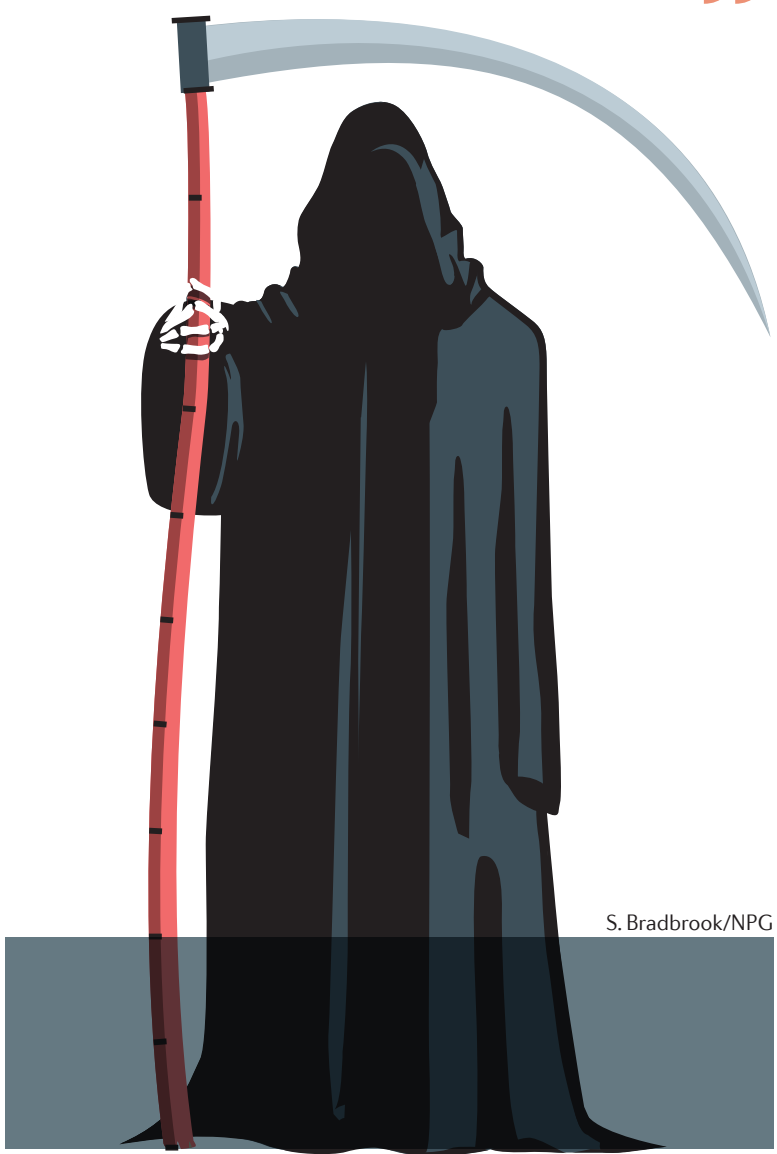

Preclinical and clinical data have shown that serum CA-125 levels are predictive of ovarian cancer risk, with high specificity. When serum CA-125 testing was used as part of a multimodal screening approach with transvaginal ultrasonography screening (USS) as a second-line test, in the 1990s, a survival benefit was noted even though the detection rate was only $65 \%$. This led to the development of the Risk of Ovarian Cancer Algorithm (ROCA) that utilizes the pattern of serum CA-125 over time to improve detection rates in population-based screening programmes to $85 \%$.

The UK Collaborative Trial of Ovarian Cancer Screening (UKCTOCS) investigators undertook this definitive randomized controlled trial to assess the effect of screening on mortality. Ian Jacobs and Usha Menon, lead authors of the study expand: "a total of 202,638 women in the UK were involved in the trial, which is one of the largest prospective interventional trials ever conducted."

Two screening approaches were used in the UKCTOCS trial: multimodal screening (MMS) that included serum CA-125 analysis (ROCA) as a first-line test, followed by USS as a secondary test for only those women deemed to be at increased risk. The second approach used USS only. Both screening approaches were compared with no screening. Women aged 50-74 years were randomized in a 1:1:2 ratio to the screening cohorts and the no screening cohort. No significant differences in mortality were observed between the arms at 7 years; however, beyond this time point (7-14 years),
MMS significantly reduced mortality. Specifically, MMS enabled detection of ovarian cancer at an earlier stage and in a greater number of patients than observed for USS or no screening group. Nevertheless, further follow up is required to fully assess the mortality reduction.

"The study is of great importance as it is the first ever to suggest that the outcome of ovarian cancer can be changed through early detection by screening. The evidence is persuasive of a mortality reduction in the order of $20 \%$, but is not yet conclusive." Jacobs continues, "UKCTOCS also confirms that of the available approaches to screening the multimodal approach interpreting CA-125 with the ROCA is the most effective and safe." The data are promising, but do not provide sufficient evidence to introduce a national screening programme for ovarian cancer in the $\mathrm{UK}$ at the present time.

How screening relates to mortality reductions through optimal treatment is also critical. To put this large screening trial in the context of treatment considerations, Steven Narod provides some additional comments: "I think there is hope that early detection from screening for ovarian cancer might actually prove to work. Ideally, we need a test that advances the diagnosis by about 12 months. Screening cannot be effective in the absence of optimal treatment. Thus, there is a need to strive for complete surgical resection of all visible cancer cells and then give intraperitoneal chemotherapy. In 2016, the best way to reduce deaths from ovarian cancer is not screening, but is through giving intraperitoneal chemotherapy."

Lisa Hutchinson

ORIGINAL ARTICLE Jacobs, I. J. et al. Ovarian cancer screening and mortality in the UK Collaborative Trial of Ovarian Cancer Screening (UKCTOCS): a randomised controlled trial. Lancet http://dx.doi.org/10.1016/S0140-6736(15)01224-6 FURTHER READING Narod, S. Can advanced-stage ovarian cancer be cured?. Nat. Rev. Clin. Oncol. http://dx.doi.org/10.1038/nrclinonc.2015.224 